\title{
Lidil
}

Revue de linguistique et de didactique des langues

\section{La mimique-gestuelle dans la description du dialogue oral en français}

Fonctions linguistiques du geste dans l'échange oral

\section{Mary-Annick Morel}

\section{OpenEdition}

\section{Journals}

Édition électronique

URL : http://journals.openedition.org/lidil/145

DOI : $10.4000 /$ lidil. 145

ISSN : 1960-6052

Éditeur

UGA Éditions/Université Grenoble Alpes

Édition imprimée

Date de publication : 1 juin 2005

Pagination : 101-117

ISBN : 2-914176-12-0

ISSN : 1146-6480

Référence électronique

Mary-Annick Morel, «La mimique-gestuelle dans la description du dialogue oral en français », Lidil [En ligne], 31 | 2005, mis en ligne le 03 octobre 2007, consulté le 01 mai 2019. URL : http:// journals.openedition.org/lidil/145 ; DOI : 10.4000/lidil.145

Ce document a été généré automatiquement le 1 mai 2019.

(C) Lidil 


\title{
La mimique-gestuelle dans la description du dialogue oral en français
}

Fonctions linguistiques du geste dans l'échange oral

\author{
Mary-Annick Morel
}

1 L'article sera fondé sur l'examen d'un des dialogues à bâtons rompus enregistrés récemment par les étudiants, sur cassette audio et sur cassette numérique vidéo, dans le cadre du séminaire « Analyse de l'oral » (Paris 3, EA 1483, 2003-2004). Une des hypothèses fondatrices de cette recherche collective est que la mimique-gestuelle est, comme l'intonation, partie intégrante de la langue - et plus précisément d'une langue particulière - et qu'on ne saurait donc parler du geste en tant que phénomène «paraverbal » ou «co-verbal », ni parler d'invariants qui transcenderaient la diversité des langues et des cultures. Les mimiques-gestuelles doivent être étudiées comme partie intégrante d'une langue dans son usage oral. Leur interprétation ne peut se faire, dans l'état actuel des recherches, que dans leur relation à un acte de parole particulier. Pour parvenir à vérifier l'hypothèse qu'il pourrait exister des fonctions stables pour les gestes dans une langue donnée, il est, bien entendu, nécessaire de procéder patiemment à des études de cas, pris les uns après les autres.

2 Mon objectif est donc ici' de mettre en lumière la démarche que nous utilisons dans cette recherche, et aussi de proposer un certain nombre de régularités, qui commencent à apparaître à travers l'analyse de nos différents enregistrements.

3 Je partirai pour ce faire d'un dialogue entre deux jeunes filles, enregistré en novembre 2003 par Edlira Çela', pour expliquer comment on y retrouve certaines des observations faites par d'autres chercheurs (voir les références bibliographiques données à la fin de l'article), et aussi certaines des hypothèses dégagées par Danielle Bouvet et moi-même (2002).

4 L'analyse d'un court extrait de ce dialogue sera présentée en prenant en compte les trois niveaux de structuration de l'échange oral: morphosyntaxique, intonatif et mimico- 
gestuel, tant du côté de la parleuse ${ }^{3}$ que du côté de celle qui écoute et réagit (l'écouteuse, dans notre jargon), qui se manifeste soit en produisant des sons, soit par des mimiques et des gestes. Mais l'essentiel de l'analyse portera sur la mimique-gestuelle.

Bref rappel

Face à la décondensation morphosyntaxique imposée par l'usage oral de la langue en français, on sait déjà (Morel et Danon-Boileau, 1998) que l'intonation a pour rôle premier d'assurer la cohésion sémantique des groupes syntaxiques et des constituants discursifs du paragraphe oral. On sait aussi que les différents paramètres de l'intonation assument chacun un rôle spécifique dans le marquage des positions interpersonnelles entre le parleur et l'écouteur, ainsi que dans la gestion de la formulation. Je me contenterai ici de rappeler que l'intensité de la voix sert à manifester le droit à la parole de chacun (colocution), que les variations de la mélodie traduisent les anticipations faites par le parleur à l'égard de la réception de ses propos et à l'égard des réactions de celui auquel il s'adresse (coénonciation).

6 Sachant que la plupart du temps le geste anticipe légèrement sur la séquence sonore, j'essaierai de montrer comment les mouvements de la tête et du regard et la gestuelle des mains s'associent aux mouvements de la mélodie et de l'intensité pour expliciter les relations coénonciatives et colocutives entre les interlocuteurs.

Quelques mots sur le dialogue analysé

Géraldine (G) a 20 ans et est étudiante dans une école de commerce. Sa mère vient de Bretagne et son père est né à Paris. Elle a vécu avec ses parents dans la région parisienne et au moment de l'enregistrement, elle habite dans un foyer de jeunes filles à Paris. Amélie (A) a 21 ans et est étudiante en Économie et Gestion. Elle a vécu jusqu'à 13 ans en Bretagne avec ses parents et depuis sept ans à Paris. Elle loge dans le même foyer que G. L'enregistrement audio et vidéo a été réalisé dans ce foyer. La conversation a duré 30 minutes, dont 6 minutes ont donné lieu à une transcription orthographique à l'écoute. De ces 6 minutes a été extrait un segment audio et vidéo de 35 secondes, pour en obtenir les tracés intonatifs avec le logiciel Praat et pour en examiner les propriétés mimogestuelles. Les deux jeunes filles se connaissent bien, elles parlent de leur week-end et de leurs amies du foyer. Pendant le passage transcrit, c'est Géraldine qui a le rôle de parleuse. Elle évoque son dernier week-end et ses amis de Barcelone qui sont venus à Paris pour quelques jours. Amélie, en bonne écouteuse qu'elle est, réagit régulièrement, comme il se doit.

Transcription orthographique du passage étudié

Géraldine $3:[. .$.$] donc jeudi soir j'avais e des amis d'Barcelone qui sont montés sur Pari::s$

Amélie 3 :-c'est vrai?

Géraldine 4 : donc voilà :, ç’a été les grandes retrouva::illes, mais e ç'a été très rapi::de [...]

Géraldine 11 : et euh §Amélie $10:$ mais où est-ce que tu les as vus§ c'est-à-dire que: on s'est retrouvés e dans PaRI::s [...]

Géraldine $14:[. .$.$] du moins on s'est tous rend/donné rendez-vous à Saint-Germai:n et$ comme j'avais un dîner ensuite eh ben j'leur avais proposé de venir $\{30\}$ le p(e)tit pro BLÈME

Amélie 14 : *à Saint-Germain* à Paris ?

Géraldine 15 : ouais j'avais u:n (h) un repa:s parce que dans mon école y a des personnes qui vienne:nt de Barcelo:::ne un p (e) tit peu de partout \{35\} §Amélie : mm§ et doncque:: 
on m:: on:: $\{20\}$ avec une amie on nous avait invitées (h) donc ce que j'avais pas prévu c'est que mes amis sont arrivés avec sept perso:::nnes ${ }^{\circ}$ donc ${ }^{\circ}$ t'imagi:::nes:: AH/ $\{80\}$ je vais PEUTÊTRE A ${ }^{\text {ppeler }}$ pour savoir si on ${ }^{\text {peut }}$ y $\mathrm{a}_{\text {ller }}\{\underline{60\}}$ §Amélie : ouais§ doncque en fin de compte ils ont bien vou::lu: doncque non c'était plutôt sympa il y avait une bonne ambia::nce:(h) d'moins bon QUE des souvenirs de Barce ${ }^{\text {lo:ne }}$ pis après la grande nos ${ }^{\text {talgie::: }}$ en rentra:nt ${ }^{\circ}$ mais e voilào ça maintenant ça y est j'suis repartie: et e

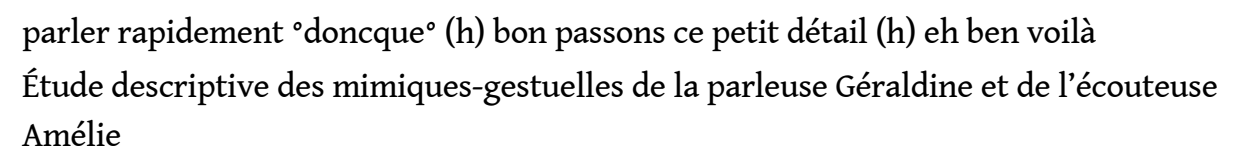

(1) ouais j'avais u:n $\{20\}$ un repa::s

Sur l'ensemble de cette séquence l'intensité varie, mais pas la mélodie. Le regard de Géraldine se dirige vers le haut sur ouais qui est en fait une réponse à la demande de précision de Amélie à Saint Germain à Paris ?; puis il se déplace vers le bas sur le premier un, accompagnant la recherche du mot juste (elle a dit préalablement j'avais un dîner). Pendant ce temps Amélie regarde Géraldine.

(2) parce que dans mon école y a des perso:nnes

Le regard de $G$ revient vers $A$ avant le ligateur parce que; sa main droite se dirige vers sa poitrine, dans un mouvement de localisation déictique par rapport à elle-même sur dans mon école, traduisant à la fois qu'elle se place de son propre point de vue et que le récit qu'elle va faire la concerne directement. Puis sur y a l'index de la main droite de $G$ se dirige vers l'espace intérieur qui la sépare de $\mathrm{A}$, son regard suit le geste de son index et sa tête s'incline un peu à droite. A la fin de la production de personnes sa tête et son regard reviennent sur Amélie.

(3) qui vienne:nt de Barcelo:::ne un $\mathrm{p}$ (e) tit peu de partout $\{35\} \S \mathrm{mm} \S$

Juste avant le qui viennent le regard de $\mathrm{G}$ quitte $\mathrm{A}$ et se dirige vers l'extérieur à droite. Simultanément sa main droite effectue un mouvement de l'extérieur vers l'intérieur, mimant en quelque sorte le sens du déplacement des personnes de Barcelone vers Paris (le verbe « venir » correspond en effet à un mouvement vers l'énonciateur). Sur le Ba- de Barcelone, la main gauche se lève et se dirige aussi vers la droite. Avant la dernière syllabe $\mathrm{du}$ nom, le regard de $\mathrm{G}$ revient sur $\mathrm{A}$ et $\mathrm{y}$ reste pendant la production de un petit peu d'partout. Les deux mains de $G$ balaient l'espace et viennent s'immobiliser face à face juste au-dessus de ses genoux, accompagnant ainsi l'idée du rassemblement de toutes ces personnes en un même lieu.

Pendant ce temps Amélie a son regard rivé sur $\mathrm{G}$; elle produit un $\mathrm{mm} 35 \mathrm{cs}$ après la fin de la séquence de $G$, immédiatement donc après la pause (35 cs) de $G$, en faisant un petit mouvement de tête de bas en haut.

(4) et doncque:: on m:: on:: $\{20\}$ avec une amie on nous avait invitées $\{20\}$

Sur toute cette séquence, qui se présente comme une glose explicative de ce qu'elle vient de dire, le regard de $\mathrm{G}$ reste sur $\mathrm{A}$, sa main gauche est dirigée aussi vers $\mathrm{A}$, comme pour lui offrir cette explication supplémentaire.

Sur on nous avait invitées A fait un mouvement de la tête de bas en haut, interprétable comme une marque de bonne écoute (équivalent à 'je te suis dans ton récit'). 
(5) donc ce que j'avais pas prévu c'est que mes amis sont arrivés avec ${ }^{\text {sept }}$ perso::nnes

Avant le donc Géraldine détourne son regard de Amélie et regarde vers le bas jusqu'à la fin du préambule ce que j'avais pas prévu. Puis son regard revient vers $\mathrm{A}$ au début du rhème c'est que mes amis sont arrivés. Mais il se dirige vers le bas, dans un mouvement d'égocentrage faisant abstraction de la situation du dialogue, au moment où elle dit sept. En même temps elle lève les sourcils et ferme les yeux, en se rémémorant le caractère incongru de la situation qu'elle aurait préféré ne pas 'voir' et dont elle pense que son écouteuse ne peut pas se l'imaginer; sur personnes elle maintient son regard vers le bas, mais elle ouvre les yeux, ses sourcils reviennent en position normale et elle hoche légèrement la tête.

Juste après la production de sept Amélie sourit, parfaitement de connivence avec son amie.

(6) donc t'imagi::::nes e:: AH/\{35\} e::: je vais peut-être

${ }^{\text {a }}$ ppeler pour savoir si on ${ }^{\text {peut }}$ y aller e $\{\underline{60}\}$ §A:

$\mathrm{Au}$ début de cette nouvelle séquence, $\mathrm{G}$ regarde toujours vers le bas, sur t'ima- elle lève les sourcils, mais sur -gines les sourcils reviennent en position normale. Puis le regard de $G$ revient vers $A$ et sa tête s'incline un peu en arrière, marquant ainsi un écart par rapport à la situation du dialogue: $G$ se projette en quelque sorte dans l'espace mémoriel de la scène qu'elle évoque. Lors de la production du $A H$ de gorge très bref, le regard de $G$ quitte A et se lève vers le haut à droite, ses yeux sont plus ouverts, comme si elle était la proie d'une illumination. Sa tête s'avance vers l'avant. Sur e je vais le regard de $\mathrm{G}$ revient sur A et y reste. Sa main droite se lève avec l'index pointé vers le haut, dans un espace situé en dehors de l'interlocution, là d'où vient l'illumination. Du point de vue de l'intonation, on note que le niveau mélodique est plus haut sur toute cette séquence de discours rapporté, qui est par ailleurs très modulée (F0 monte sur les deux points les plus importants : le $a$ de appeler et le verbe peut). Puis avec peut-être appeler, le buste et la tête de G s'inclinent un peu vers l'avant, dans un mouvement de sollicitation en direction de A (signifiant en quelque sorte 'tu ne peux que partager ma réaction, tu aurais fait comme moi'). Sur pour savoir si on peut les deux index sont tendus vers le haut; ils pointent ensuite vers l'avant sur $y$ aller, dans un mouvement partant du parleur et dirigé vers l'extérieur (suivant là encore, comme pour viennent, le sens en langue de aller). Le regard de $\mathrm{G}$ est toujours sur A, ses yeux sont plus largement ouverts, puis son buste revient vers l'arrière.

De son côté Amélie lève les sourcils sur je vais, et son regard décroche de $G$ pour se diriger vers le haut, dans un mouvement mimétique de celui de $G$ au moment où elle a dit $A H$. Son regard revient ensuite vers $G$ sur le peut de si on peut, et 30 cs après elle manifeste sa bonne écoute par un ouais accompagné d'un hochement de tête bas-haut.

(7) doncque en fin de compte ils ont bien vou::lu:::

Sur le ligateur doncque la tête de $\mathrm{G}$ se déplace un peu en avant légèrement à gauche, dans un mouvement de sollicitation à l'égard de $\mathrm{A}$; mais le regard reste sur A. Puis il ne se passe rien d'autre sur le plan gestuel. Seul l'allongement des deux syllabes de voulu souligne le soulagement qui accompagne cette issue favorable.

(8) doncque non c'était plutôt sympa il y avait une bonne ambia::nce:\{60\}

A nouveau le regard quitte A sur le ligateur doncque et se dirige vers le bas; puis la tête s'incline un peu vers l'avant droite au moment du non, qui est prononcé en plage mélodique plus haute, marquant le statut cataphorique de ce non. Puis sur c'était plutôt un 
mouvement de la tête de droite à gauche accompagne la modalité appréciative nuancée de plutôt et explicite le contenu du non, signifiant quelque chose comme « contrairement à ce que laissaient présager mes propres prédictions sur le côté désagréable de la situation ». Sur il y avait, la tête de G s'incline à droite, le buste va vers l'avant. La syllabe finale de ambiance est allongée soulignant qu'il y aurait sans aucun doute plus à dire sur la qualité de l'ambiance.

Amélie hoche la tête en signe d'acquiescement.

(9) d'moins bon QUE des souvenirs de Barce ${ }^{\text {lo:ne }}$ donc

Le ligateur complexe d'moins bon donné en mélodie basse marque la fin de ce commentaire appréciatif, pendant que le regard dirigé vers le bas accompagne cette phase d'évaluation égocentrée. Sur QUE doté d'une forte intensité, le regard de $G$ revient vers A. Une montée mélodique caractérise la dernière syllabe allongée de Barcelo::ne marquant ainsi l'exclamation. Le donc final bas et plat assure la valeur conclusive, récapitulative de cette glose.

(10) pis après la grande nostalgie::: en rentra:nt ${ }^{\circ}$ mais e voilà

Le ligateur pis après a ici sa pleine valeur temporelle, il anticipe sur le gérondif en rentrant en introduisant le sentiment qui a envahi $\mathrm{G}$ à son retour à Paris. Un réhaussement de l'intensité et un allongement de la durée sur les trois syllabes du mot nostalgie et une remontée de F0 sur la dernière syllabe -gie attirent l'attention sur le haut degré qualitatif de la notion évoquée. Le changement de cadre spatio-temporel n'est pas totalement explicité dans la séquence exclamative elle-même la grande nostalgie; mais ce sont les mimiques-gestuelles qui le signifient : le regard part vers le haut, il fait un mouvement circulaire deux fois, accompagné par la tête, comme si yeux et tête mimaient un mouvement de désarroi aboutissant à tourner en rond. A la fin de la séquence en rentrant la tête et le regard reprennent leur position vers A. Sur la clôture mais e le regard de G est revenu vers A, dotant le voilà, prononcé en mélodie basse et descendante, d'une valeur déictique en direction de A ('je voudrais que tu voies comme moi').

Amélie lève les sourcils et ouvre un peu plus les yeux, ce qui traduit sa surprise: l'expression de son visage accompagne l'émotion qui jalonne le récit de G. Puis sourcils et yeux reprennent leur état « normal » à la clôture.

À la fin de la séquence, $G$ ne fait pas d'autres gestes; A n'en fait pas non plus. On peut conclure de cette absence de geste que « un ange passe », mais aussi que 'tout est bien qui finit bien'.

(11) ça maintenant ça y est j'suis repartie et e::

Sans pause aucune, $\mathrm{G}$ revient brusquement au hic et nunc de la situation du dialogue en cours avec maintenant. Cette séquence est toutefois ininterprétable en elle-même (j'suis repartie: on se demande "où?»), d'où l'amorce de réaction de A mais ça fait, qui va déclencher le commentaire explicatif de $\mathrm{G}$. Sur le et e final $\mathrm{G}$ laisse chuter l'intensité.

(12) Amélie 15 : *mais ça fait/*

48 Ayant perçu l'ambiguïté du propos de $\mathrm{G}$ et chute de l'intensité à la fin sur et e::, A manifeste une velléité d'intervention en disant dans un débit très rapide mais ça fait en plage intonative haute, mais sans grande intensité. Les propriétés intonatives montrent qu'il s'agit plus d'une marque d'écoute et d'intérêt que d'un véritable désir de s'emparer du droit à la parole. Le regard de A est d'ailleurs resté fixé sur G.

(13) Géraldine 16 : j’ai parlé très rapideme:nt e $\{20\}$ e:: 
$50 \quad G$ du coup hausse l'intensité pour réassurer son droit à la parole et expliciter davantage son commentaire sur sa façon de parler. A se tourne alors vers la caméra (tenue par Edlira) comme pour la prendre à témoin et sourit. Sur j'ai parlé très rapidement, $\mathrm{G}$ se tourne aussi vers la caméra (tête et regard conjointement), elle fait un mouvement de la main droite haut-bas dans l'espace devant elle, comme pour apaiser ce débit intempestif.

51 (14) parce que c'est ma e: mon habitude de parler rapidement doncque $\{100\}$

$52 \mathrm{G}$ retourne ensuite vers A et maintient son regard sur elle. C'est au tour de la main gauche de faire le mouvement en deux temps haut-bas devant elle, d'incitation adressée à ellemême à ralentir le débit.

Synthèse des mimiques-gestuellesRécapitulation de la valeur des mouvements de la tête et du regard

53 Concernant les mouvements de la tête et du regard, nous rencontrons dans ce corpus un certain nombre de régularités.

Mouvements concernant les interlocuteurs

54 - La tête et le regard de la parleuse ne restent pas rivés sur l'écouteuse, mais au contraire la quittent à des moments précis, à savoir en début de préambule (1) ouais (2) y a (5) donc ce que j'avais pas prévu, (6) AH, (8) doncque non, (10) pis après.

55 - Tête et regard de la parleuse reviennent au moment de la production de sa position différenciée, avant la fin du rhème donc (2) parce que e (5) c'est que mes amis sont arrivés avec, (6) je vais peut-être appeler pour savoir si on peut y aller, (8) que des souvenirs de Barcelone donc (9) en rentrant 'mais e voilà.

56 - Le regard de la parleuse reste, en revanche, sur l'écouteuse pendant toute la séquence quand il s'agit d'un énoncé de glose, d'un commentaire explicatif, n'impliquant pas de modification du point de vue : (2) parce que dans mon école, (3) un petit peu d'partout, (4) et doncque avec une amie on nous avait invitées.

Mouvements dans une autre direction que celle de l'interlocuteur

57 - Le regard vers le bas est lié (dans ce corpus, comme dans beaucoup d'autres) à la localisation dans la sphère personnelle de la parleuse: lors de la recherche d'un mot (1) j'avais un un repas; et à son implication forte dans la situation évoquée: devant le caractère incongru de la situation (5) ce que j'avais pas prévu + sept personnes donc t'imagines, ou dans son appréciation de l'atmosphère du dîner $(8+9)$ doncque non c'était plutôt sympa il $y$ avait une bonne ambiance d'moins bon.

58 - Le regard vers le haut ${ }^{5}$ accompagne dans ce corpus un fait venant de l'extérieur, qui n'était pas spécialement programmé par le parleur-narrateur :

- le ouais préalable au début du récit, donné en réponse à la demande de précision pertubatrice de A : (1) ouais < j'avais un repas >

- le discours rapporté (fictif) qui explicite la seule chose à faire pour redonner de la normalité à cette situation inimaginable : (6) $\mathrm{AH}$

- l'évocation de son état d'esprit au retour de Barcelone : (9) la grande nostalgie

59 Dans les deux cas (regard vers haut et regard vers le bas), la référence temporelle des événements évoqués est décrochée du hic et nunc de la situation.

60 - Le regard vers l'extérieur à droite indique, tout comme la main, la localisation déictique d'un lieu autre que celui de l'interlocution, à savoir ici Barcelone: (3) qui viennent de Barcelone, et, quand il se dirige vers le haut, la localisation de la source de l'inspiration subite de téléphoner (6) $\mathrm{AH}$. 
Mouvements spécifiques de la tête et du regard iconique de manifestation de désarroi, et de circularité des souvenirs: (10) la grande nostalgie.

Mouvements du regard associé à d'autres indices

62 comme dans beaucoup d'autres) il a le plus souvent pour fonction de préciser qu'il s'agit de référents bien circonscrits par le parleur, même si l'expression morphosyntaxique (article indéfini des) ne le spécifie pas : (2) y a des personnes.

63 - Le regard vers le bas associé au haussement des sourcils' et à la fermeture des yeux traduit l'indicible de la situation, son caractère inimaginable qu'on ne peut se rémémorer qu'en fermant les yeux sur le monde extérieur : (5) sept < personnes >.

La direction du mouvement des mains est également très cohérente dialogue partagé par les deux interlocutrices; vers la droite, il localise, tout comme le regard, Barcelone.

- Le mouvement des mains vers l'intérieur, entre les interlocutrices, ramène à l'espace partagé par les deux interlocutrices, celui (large) de Paris : (3) qui viennent de Barcelone, ou celui (restreint) du dialogue, à savoir :

- en direction de la parleuse elle-même, de sa poitrine : la localisation déictique est faite par rapport à elle-même, pour marquer que c'est son point de vue personnel qui est en jeu : (2) j'avais un repas

- vers l'interlocutrice : (4) avec une amie on nous avait invitées

- vers le haut, c'est à dire, dans ce dialogue, là d'où vient l'inspiration subite : (6) je vais ; puis avec les deux index pointés vers le haut : (6) pour savoir si on peut.

- Le déplacement des mains qui balaient l'espace et viennent s'immobiliser face à face juste au dessus des genoux du parleur a, en revanche, une fonction plus illustrative, mimant le mouvement de déplacement de l'extérieur vers le parleur associé au sens en langue du verbe venir : (3) viennent, ainsi que le rassemblement : (3) un petit peu d'partout; alors que le mouvement partant du parleur vers l'extérieur est associé à celui du verbe aller.

Mouvements de la tête et du buste correspondent à ce que Montagner (1988: 245), cité par Danielle Bouvet (2001:20), appelle «mouvement de sollicitation » (émanant du parleur), de recherche d'approbation et de partage de la part de l'écouteur.

Le « ballet de la parole » est bien illustré dans ce dialogue

L'écouteuse, par la connaissance qu'elle a des règles de bon fonctionnement du dialogue en français, utilise à bon escient toutes les manifestations de bonne écoute qui sont à sa disposition. Elle recourt donc alternativement (plus rarement en même temps) aux indices suivants.

Elle lève les sourcils au moment du discours rapporté direct de $G(6)$ ah je vais peut-être appeler..., et après l'énoncé du mot nostalgie (10).

Elle fait des oscillations verticales de la tête après une syllabe en mélodie montante dans le discours de $G$ et maintient, ce faisant, son regard sur G, notamment après les mots (4) 
invitées < avec une amie on nous avait invitées >, (8) sympa < c'était plutôt sympa > et bonne ambiance $<$ il y avait une bonne ambiance $>$.

Elle produit des marques sonores (3) $\mathrm{mm}$ et (6) ouais respectivement $35 \mathrm{cs}$ et $30 \mathrm{cs}$ après la fin d'une séquence sémantiquement cohérente de $\mathrm{G}(3)<$ qui viennent de $>$ Barcelone un petit peu d'partout, (6) je vais peut-être appeler pour savoir si on peut $<y$ aller $>$, et cela lorsque le regard de la parleuse est revenu dans sa direction.

Son sourire apparait après avoir entendu (5) sept marquant ainsi sa connivence devant l'insolite de la situation.

73 Elle déplace son regard dans la même direction que le regard de la parleuse, au moment où celle-ci opère une modification dans le cadre spatio-temporel, lors de l'évocation du coup de fil : (6) je vais peut-être appeler pour savoir si on peut $<y$ aller $\rangle$.

Elle fait une tentative pour apporter son aide sonore lorsqu'elle perçoit une difficulté dans la cohérence des propos et la gestion de la suite par G : (12) mais ça fait, mais elle le fait sans hausser l'intensité et en regardant $G$, signes qui témoignent qu'elle n'a pas vraiment envie de lui usurper son droit à la parole.

Cas de superposition des indices des trois plans

75 L'intérêt particulier de ce court extrait provient de ce que la parleuse relate une situation banale (invitation de personnes amies à un repas où on est déjà soi-même invité), mais qui par le nombre des personnes est subitement devenue ingérable, incongrue. D'où la mise en scène du récit par le recours aux différents plans d'indices, qui, remarquons-le une fois de plus, ne se superposent généralement pas, mais apparaissent comme un choix laissé au libre arbitre du parleur dans le dialogue à bâtons rompus (ce serait bien évidemment à vérifier dans des situations différentes d'échange). De ce fait, dans les moments où ils apparaissent conjoints, le discours n'en est rendu que plus saillant.

La superposition simultanée des indices des trois plans qui accompagne l'expression de la solution trouvée à cette situation inextricable le montre. Le discours intérieur rapporté (voir (6)) qui se présente comme l'événement-clé pouvant seul conduire au dénouement est ainsi « réhaussé » sur tous les plans. Il présente :

- une interjection initiale brève et forte $A H$

- une plage mélodique haute et multiples variations de F0 : je vais peut-être appeler pour savoir si on peut y aller

- une avancée du buste

- le regard porté vers le haut sur AH

- le pointage de l'index vers le haut également.

77 Alors que le dénouement intermédiaire, de peu d'intérêt en vérité, après cette tension dramatique: (7) doncque en fin de compte ils ont bien voulu, n'est marqué que par deux indices faibles, fugitifs et sans durée : léger déplacement de la tête vers l'avant gauche et allongement des deux syllabes de voulu. Quant à la ponctuation finale de cette anecdote, elle est, elle aussi, très peu marquée, le seul indice gestuel étant le mouvement de la tête vers le haut sur mais e voilà.

78 C'est donc à partir d'un grand nombre de descriptions sur des extraits minuscules du genre de celui que je viens de présenter que nous espérons préciser les hypothèses concernant la place de la gestuelle dans le dialogue en français, et son statut dans la description de la langue. 


\section{BIBLIOGRAPHIE}

BOUVET, D. (2001) : La dimension corporelle de la parole : les marques posturo-mimo-gestuelles de la parole, leurs aspects métonymiques et métaphoriques, et leur rôle au cours d'un récit, Louvain, Peeters, Collection de la Société de linguistique de Paris.

BOUVET, D., DANON-BOILEAU, L., MOREL, M.-A. (1998) : Se parler, c'est aussi se regarder et se donner à voir, in Orage'98, Oralité et Gestualité. Communication multimodale, interaction, L'Harmattan, 569-576. BOUVET, D., MOREL, M.-A. (2001) : Les réalisations formelles de la coénonciation : coocurrence et distribution des indices des trois plans : morphosyntaxique, intonatif, posturo-mimo-gestuel, in Orage'01, Oralité et Gestualité. Interactions et comportements multimodaux dans la communication, L'Harmattan, 482-487.

BOUVET, D., MOREL, M.-A. (2002) : Le ballet et la musique de la parole. Le geste et l'intonation dans le dialogue oral en français, Paris-Gap, Ophrys, Bibliothèque de Faits de Langues. [abrégé BM] BOYER, J. (1998) : Effets de la simultanéité de production entre gestes iconiques ou métaphoriques et contenus verbaux ou intonatifs, in S. Santi, I. Guaitella, C. Cavé et G. Konopczynski (dir.), ORAGE'98, Paris, L'Harmattan, 329-336.

DANON-BOILEAU, L., MOREL, M.-A. (2003) : Le locuteur vicariant, in J.-M. Merle coord., Le sujet, ParisGap, Ophrys, Bibliothèque de Faits de Langues, 235-246.

DEMERS, M. (dir.) (2003) : Registre et voix sociale, Québec, Éditions Nota Bene.

GARDNER, R. (2001) : When listeners talk. Response tokens and listener stance, Amsterdam/Philadelphia, John Benjamins Publ. Comp.

GULLBERG, M. (1998) : Gestures and Speech in Second Language Interaction, in Oralité et Gestualité (Orage'98), S. Santi, I. Guaitella, C. Cavé et G. Konopczynski (dir.), Paris, L'Harmattan, 641-646.

LAKOFF, G., JOHNSON, M. (1980) : Metaphors we live by, Chicago, University of Chicago Press (trad. Fr. (1985), Les métaphores dans la vie quotidienne, Paris, Ed. de Minuit).

MONTAGNER, H. (1988) : L'attachement, les débuts de la tendresse, Paris, Odile Jacob.

MOREL, M.-A., DANON-BOILEAU, L. (1998) : Grammaire de l'intonation. L'exemple du français oral, Bibliothèque de Faits de langues, Paris-Gap : Ophrys. [abrégé GI]

NOBE, S. (2001) : On gestures of foreign language speakers, in Oralité et Gestualité (Orage'01), C. Cavé, I. Guaïtella, S. Santi (dir.), Paris, L'Harmattan, 572-575.

SANTI, S., GUAitella, I., CAVÉ, C., KONOPCZYNSKI, G. (dir.) (1998), Oralité et gestualité. Communication multimodale, interaction, Actes du colloque ORAGE'98, Paris, L'Harmattan.

TABENSKY, A. (1998) : Gestes déictiques et métaphoriques dans l'interaction. Une étude comparative transculturelle, in S. Santi, I. Guaitella, C. Cavé et G. Konopczynski (dir.), ORAGE'98, Paris, L'Harmattan, 393-398.

TABENSKY, A. (2001) : Le pointage en anglais, en espagnol et en français, in C. Cavé, I. Guaïtella, S. Santi (dir.), Oralité et Gestualité (Orage'01), Paris, L'Harmattan, 586-590. 


\section{ANNEXES}

Glossaire

Coénonciation: La façon dont celui qui parle (le parleur) envisage la réception de son discours par celui auquel il s'adresse (l'écouteur) est explicitée par les variations de hauteur mélodique. L'articulation à la pensée prêtée à l'autre (connaissances partagées, convergence du point de vue, objections possibles...) et l'anticipation de ses réactions relèvent de la coénonciation ; une mélodie montante anticipe un partage de consensus, alors qu'une mélodie descendante accompagne une prise de position égocentrée, sur un point dont l'énonciateur pense que l'écouteur-coénonciateur ne le partage pas. (BM 13)

Colocution : La prise en compte du droit à la parole de chacune des parties prenantes du dialogue et l'anticipation d'une éventuelle prise de parole du colocuteur (écouteur) sont gérées par les variations d'intensité. Le colocuteur n'est pas la personne physique à qui l'on parle, mais la façon dont on envisage son droit à parler. (BM 12)

Paragraphe: Le paragraphe oral se définit par des indices intonatifs de démarcation finale, à savoir la chute conjointe de la mélodie et de l'intensité.

Parleur et écouteur: Les participants d'un dialogue sont désignés par les termes de « parleur » et d'« écouteur » (Danon-Boileau et Morel, 2003), qu'il faut entendre dans leur acception agentive de nom dérivé de verbe avec un suffixe marquant l'agent de l'action (l'écoute étant ainsi conçue comme une activité à part entière). On s'attend, bien évidemment, à rencontrer une exploitation des variations intonatives et de la mimiquegestuelle plus abondante dans le discours du parleur que dans celui de l'écouteur (Bouvet, 2001 ; Nobe, 2001). Il n'en reste pas moins que les manifestations sonores de l'écouteur ( $\mathrm{mm}$, ouais, ah bon...), tout comme ses réactions mimico-gestuelles, sont, elles aussi, indispensables au bon fonctionnement du dialogue.

Préambule : Une des propriétés spécifiques du français est la décondensation maximale du préambule. Il peut en effet présenter une succession de sous-constituants de fonction différente, donnés dans un ordre contraint, qui servent à mettre en place la coénonciation en découpant progressivement les supports de prédication et les zones de sens : < ligateur + point de vue + modus dissocié + cadre + support lexical disjoint >. (GI 37-44, BM 26)

Rhème : C'est le constituant où le parleur tient à marquer une position différenciée en fonction des attentes qu'il prête à l'écouteur.

\section{NOTES}

1. Les limites imposées par le format de cet article m'empêchent de mentionner tous les travaux actuels sur la gestuelle dans le dialogue. L'originalité de la démarche de l'EA 1483 me semble résider 1) dans la prise en compte conjointe des indices fournis par la syntaxe, l'intonation et la mimique-gestuelle, 2) dans l'attention portée en priorité aux réactions sonores et mimico-gestelles de celui auquel le discours est adressé (l'écouteur).

2. Étudiante actuellement inscrite en Doctorat Sciences du langage à Paris 3 (ED 268), titre de la thèse : Formes et fonctions du couplage de la mimique-gestuelle et de l'intona tion 
dans le dialogue. L'exemple des déictiques dans un dialogue oral en albanais. Confrontation avec le français.

3. Un glossaire du métalangage utilisé est donné à la fin de l'article. Mention portée en priorité aux réactions sonores et mimico-gestuelles de celui auquel le discours est adressé (l'écouteur).

4. Conventions de transcription : , pause moyenne de 40 à 60 centise condes (cs)/, pause supérieure à $60 \mathrm{cs} /\{40\}$ durée de la pause silencieuse en centisecondes/x: ou x::

allongement de la durée de la voyelle/(h) reprise de respiration audible (environ $20 \mathrm{cs}$ )/e ou e:: 'euh' d'hésitation $/{ }^{\circ} \mathrm{xxx}^{\circ}$ incise (décrochement intonatif en plage basse)/* $\mathrm{xx}^{*}$ débit très rapide/§m $\S$ ou $\S \mathrm{mm} \S$ marque d'écoute de l'interlocuteur/en petites capitales : syllabe(s) prononcée(s) avec plus d'intensité/les passages soulignés notent la superposition des paroles des interlocuteurs. Les < > encadrent un fait audible < rire > ou une séquence ambiguë/les syllabes en exposant traduisent une montée mélodique, et en indice une descente. F0 désigne le fondamental de la voix dont les variations constituent la mélodie.

5.« Le plus est en haut » comme le rappelle Danielle Bouvet (2001:17) citant Lakoff et Johnson (1980); mais on peut aussi « lever les yeux au ciel » face à quelque chose qu'on ne saurait accepter.

6. Il nous semble que la direction droite-gauche n'est pas figée dans une valeur propre. Elle dépend du libre choix du parleur.

7. Le haussement des sourcils est souvent lié à une intensité : quantitative (1) sept, ou qualitative (6) t'imagines.

\section{AUTEUR}

MARY-ANNICK MOREL

Paris 3, EA 1483, marym@ext.jussieu.fr 